\title{
Analysis of the overlearning-extinction effect in honeybees
}

\author{
AKIRA SHINODA \\ Gakushuin University, Tokyo, Japan \\ and \\ M. E. BITTERMAN \\ University of Hawaii, Honolulu, Hawaii
}

\begin{abstract}
In previous experiments with individual honeybees that visited the laboratory regularly to take sucrose solution from a target set on the shelf of an open window, the overlearning-extinction effect was found for high concentrations of sucrose but not for low. The purpose of the present experiment was to examine the possibility that declining resistance to extinction in the course of prolonged training with a high concentration of sucrose could be explained in terms of increasing nutritive level. Three groups of animals were extinguished on a distinctive target, one group after 6 visits to the target, a second after 18 visits to the target, and a third after 6 visits to the target that were interspersed among 12 visits to a different target. More rapid extinction in the second group than in the third, which had fewer training visits to the extinction target than the second but the same total number of training visits, rules out an explanation in terms of nutritive level and points instead to a frustration-like process evidenced also in earlier work on incentive contrast.
\end{abstract}

The overlearning-extinction effect appeared very early in a series of comparative experiments with honeybees that have continued to yield a wide array of phenomena familiar from the study of learning in vertebrates (Couvillon \& Bitterman, 1980). After 1, 2, 3, 6, or 12 visits to a target containing sucrose solution, free-flying foragers returning to the target from the hive found water instead of sucrose, and their persistence in responding to the target (resistance to extinction) was measured on the assumption that it would provide a useful measure of the strength of association between the target and the sucrose. The results showed increasing resistance as the number of training visits increased from 1 to 6 , followed by a significant decline. The effect was replicated immediately under the same conditions and later in several other experiments (Couvillon \& Bitterman, 1984), where it was found to depend, as it does in rats (Ison \& Cook, 1964; Traupmann, 1972), on magnitude of reinforcement. Honeybees trained with $50 \%$ sucrose solution showed the effect, but honeybees trained with $20 \%$ sucrose did not.

Two alternative interpretations of these results suggested themselves. The most intriguing was that we had come upon something akin to frustration in vertebrates (Amsel, 1958, 1962). Performance in extinction might be thought to have been disrupted by emotional response to an unrealized expectation of food that was very strong

This research was supported by Grant BNS 83-17051 from the National Science Foundation. Requests for reprints should be addressed to M. E. Bitterman, Békésy Laboratory of Neurobiology, 1993 EastWest Road, Honolulu, HI 96822. after long training with sucrose of high concentration. A more mundane possibility was that what we were seeing was only the dependence of resistance to extinction on nutritive level or hunger drive, another familiar vertebrate phenomenon (Perin, 1942). Unpublished work in this laboratory by $\mathbf{N}$. Satake and Bitterman with bees harnessed as for proboscis conditioning (Bitterman, Menzel, Fietz, \& Schäfer, 1983) had established that sucrose is taken up continuously from the social stomach, and it might be, therefore, that resistance to extinction declined in the course of prolonged training with sucrose of high concentration simply because the nutritive level of the animals increased.

For independent evidence, we turned to an experiment on successive negative contrast, another important phenomenon of vertebrate learning dealt with by frustration theory. Free-flying foragers were permitted a series of visits to $50 \%$ sucrose on one target, $A$, alternating with visits to $20 \%$ sucrose on a distinctly different target, B, and then, after a final visit to $B$, they were tested with $20 \%$ sucrose either on A (contrast group) or on B (control group). The contrast animals showed considerable disturbance before settling down to uninterrupted feeding on $A$, which could readily be attributed to unrealized anticipation (Couvillon \& Bitterman, 1984).

In the experiment now to be reported, we looked again for the overlearning-extinction effect under conditions in which nutritive level was controlled. Three groups of honeybees were extinguished on target $A$ after 6 visits to $A, 18$ visits to $A$, or 6 visits to $A$ interspersed among 12 visits to $B$, a discriminably different target. The criti- 
cal feature of the experiment was the third group, which had the same number of visits to $A$ as the first group but the same total number of visits, and hence the same nutritive level in extinction as the second. Another noteworthy feature of the present experiment was that the measurement of performance in extinction was somewhat more elaborate than it had been in the earlier experiments. Not only were contacts with the target observed by the experimenter as before, but dips into a well that contained sucrose solution during training and water during extinction were recorded with a photodetector-a technique invented by Grossmann (1973).

\section{METHOD}

\section{Subjects}

The subjects were 24 honeybees, all experimentally naive. They were recruited from our own hives, which were set up in the vicinity of the laboratory.

\section{Apparatus}

Recessed in the gray shelf of an open laboratory window was a Plexiglas food well, $.65 \mathrm{~cm}$ in diameter, into which the bees dipped for sucrose solution. The food well was much like that described by Sigurdson (1981), except that its bottom was sealed and the sucrose solution was introduced from above. At the mouth of the well, just below the level of the shelf, were a small infrared light source and a photodetector for registering the dipping response. On the shelf, encircling the well, was a removable plastic annulus, $6 \mathrm{~cm}$ in outside diameter, called a target. The plastics used for this purpose were of two colors, nominally green and gray (the same gray as the shelf), which were known from our previous work to be readily discriminable by the bees; because of its dark contour, the gray annulus also was readily discriminable from its gray background. Contacts of the animal with the target were observed by the experimenter, who pressed a hand-held switch as each occurred, and these events, together with entrances into and exits from the food well monitored by the photodetector, were tracked in real time by a microcomputer.

\section{Procedure}

As usual, the work was done with marked individual foragers, each of which was trained and extinguished in a single session. An animal would be picked up at a feeding station, which was equipped with a large jar of $10 \%-12 \%$ sucrose solution, and assigned to one of three experimental groups of 8 animals each in planned quasirandom order. The first step was to set the animal down at the food well on target $A$ (the distinctive green target), which was filled to the brim with $50 \%$ sucrose solution. While it was drinking, the animal was marked with a spot of colored lacquer; when it was replete, it was permitted to leave for the hive. Typically, the animal would return to the window of its own accord in a few minutes, but, if it did not, it was picked up again at the feeding station, where it usually could be found, and carried back to the target. The pretraining ended with the first spontaneous return of the animal to the target, which was counted as the 1st training visit. On the next 3 visits, the level of the sucrose solution in the food well was reduced progressively until it fell below the level of the photodetector, requiring the animal to dip into the well and so to activate the detector in order to take the food. The subjects of Group 6 were permitted 6 visits to the food well before extinction, and the subjects of Group 18 were permitted 18 visits. The subjects of Group 612 also had 18 visits, on 6 of which (visits $1,4,8,11,15$, and 18) target $A$ was used, and on the rest of which target $B$ (the less distinctive gray target) was used. After each training visit, while the subject was at the hive, the sucrose solution remaining in the food well was removed, the well, the target, and the surroundings were cleaned, and preparations for the next visit were made. On the 7th visit for each animal of Group 6 and the 19th visit for each animal of the other two groups, target $A$ was in place, the food well contained tap water instead of sucrose solution, and performance was studied (contacts and dips recorded) in the 10-min period after arrival.

\section{RESULTS}

The performance of the three groups in extinction is plotted in Figure 1 in terms of mean cumulative number of contacts with the target in successive 30-sec intervals. The curves for Groups 6 and 18 are very much like those of previous experiments (Couvillon \& Bitterman, 1980, 1984), showing substantially lower resistance to extinction after the larger number of training visits. The interesting new result is that the resistance of Group 6-12 was less than that of Group 6 but greater than that of Group 18; that is, resistance to extinction on target $A$ was reduced by 12 additional visits to target $B$, but reduced still more by 12 additional visits to target $A$. Analysis of variance yields a significant difference between Groups 6 and 6-12 $[F(1,14)=5.63, p=.0325]$. It also yields a significant difference between Groups 6-12 and 18 $[F(1,14)=6.92, p=.0198]-\mathrm{a}$ target-specific overlearning-extinction effect.

In Figure 2, performance in extinction is plotted in terms of the mean cumulative number of dips into the food well in successive $30-\mathrm{sec}$ intervals. The pattern of results

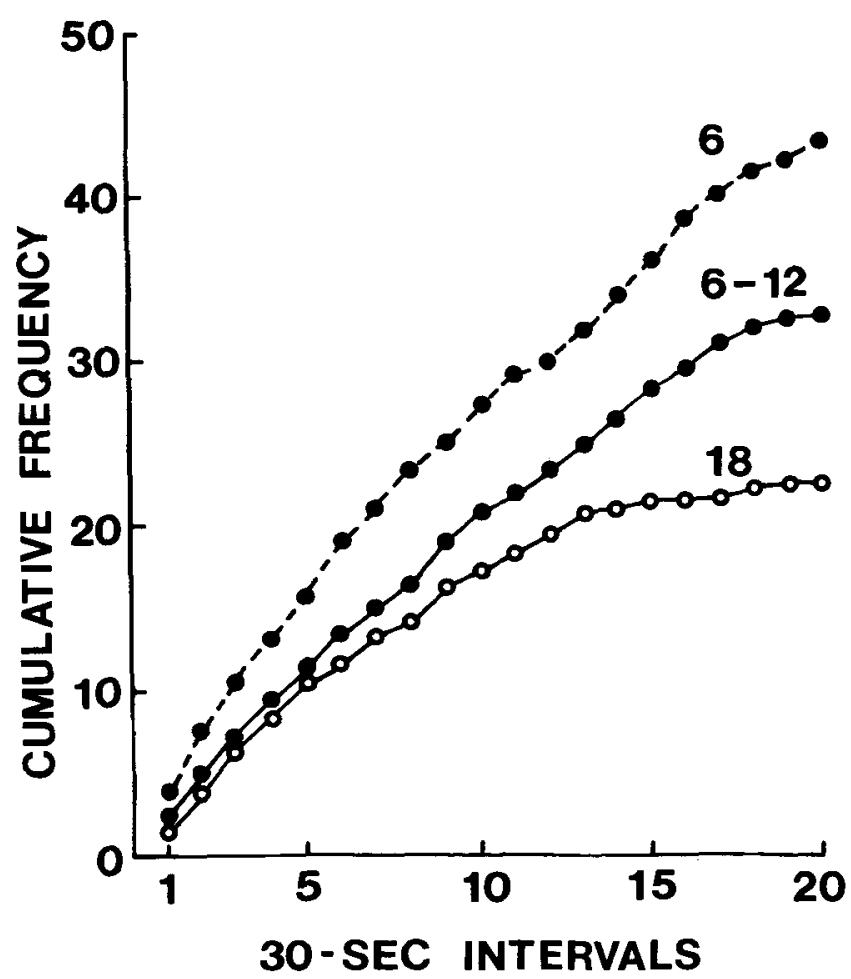

Figure 1. The course of extinction in the three groups plotted in terms of cumulative number of contacts with the target in successive 30-sec intervals. 


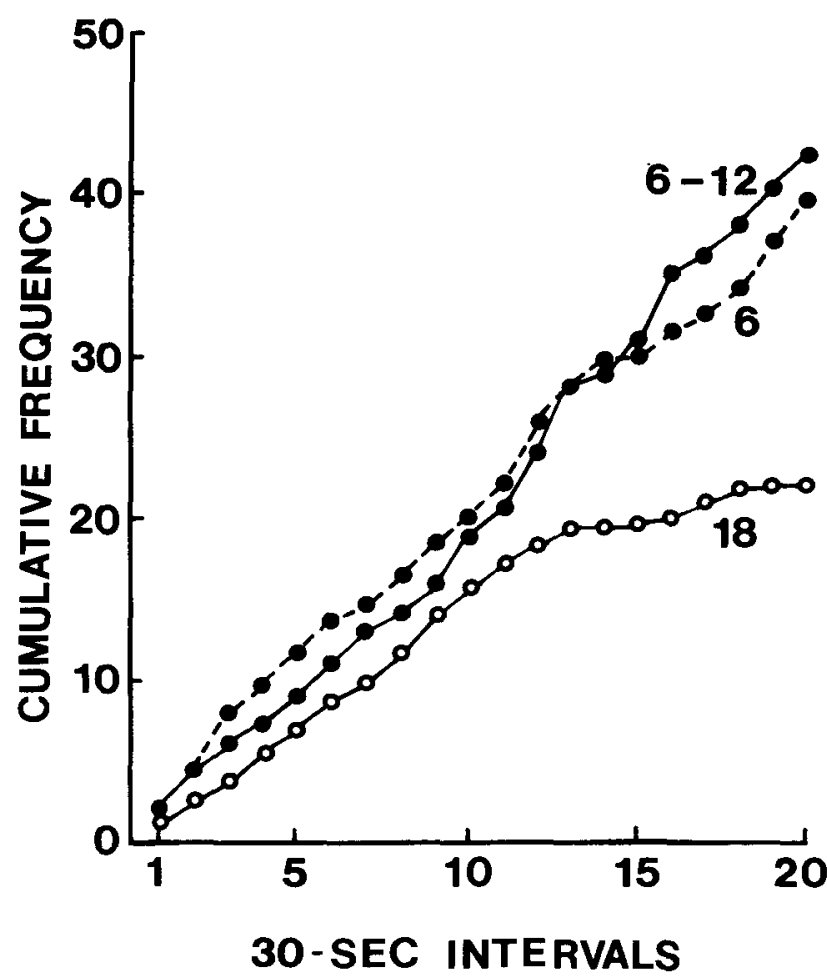

Figure 2. The course of extinction in the three groups plotted in terms of cumulative number of dips into the food well in successive 30-sec intervals.

for dips is not the same as for contacts in that the resistance of Group 6-12, although greater than that of Group 18, was not reduced by the added visits to target $B$. Analysis of variance shows no significant difference between Groups 6 and 6-12 $(F<1)$, but does show a significant difference between Groups 6-12 and $18[F(1,14)=6.64$, $p=.0219]$-the target-specific overlearning-extinction effect. A pattern of results like that for the number of dips appeared also for time between the first and last dips occurring in the 10-min extinction period. The median times were $554 \mathrm{sec}$ for Group 6, $548 \mathrm{sec}$ for Group 6-12, and $287 \mathrm{sec}$ for Group 18. Although the times for the animals of Group 6-12 were much the same as those for the animals of Group 6, Fisher's exact test showed the times for Group 6-12 to be significantly longer than those for Group $18(p=.0047)$.

\section{DISCUSSION}

As measured in terms of the number of contacts with target A, Group 6-12 (which had 12 supplementary visits to target B) showed less resistance to extinction than did Group 6 (which had only 6 visits to target A). The difference may be attributable either to reduced hunger in Group 6-12 or to a stronger general expectation based on contextual conditioning and consequently greater frustration. Just why such a difference between these two groups did not appear in resistance to extinction as measured in terms of the number of dips is not clear. One possibility is that the performance ceiling is lower for the dipping measure than for the contact measure. A moment's thought will show, of course, that the two measures cannot be perfectly correlated; an animal may dip repeatedly during a single landing on (contact with) the target, and it may contact the target repeatedly for a time without dipping at all. Whatever the reason for the discrepancy, both measures do show less resistance to extinction on target $\mathbf{A}$ in Group 18 than in Group 6-12. Since Group 18 had more training visits to target $A$ than did Group 6-12 but the same total number of training visits and hence the same nutritive level, its lower resistance to extinction may be attributed to what is described in the vertebrate literature as stronger expectation and greater consequent frustration.

However useful these borrowed metaphors may seem in the early stages of our investigation, they do not take us very far, and actually may be quite misleading in their implication of functional commonality. As has been noted before (Couvillon \& Bitterman, 1984), the fact that the various paradoxical reward effects that gave rise to frustration theory have not been found in descendants of older vertebrate lines points strongly to convergence. It may be well, therefore, to concentrate for a time on the honeybee data alone and to look for the simplest interpretation of them, which may turn out to be less elaborate than that required by the vertebrate data. A reasonable place to begin is with an association between target and sucrose that tends to produce approach to the target. Although the animals may prove, in the end, to learn about degree of sweetness (associating one target with the taste of a $20 \%$ sucrose solution, for example, and another with the taste of a $50 \%$ sucrose solution), it is sufficient for the moment to assume that associations established when different concentrations are used differ in strength rather than in signification-that associative strength grows as a function of the number of reinforced trials to an asymptote that increases with the magnitude of reinforcement (Hull, 1943).

To account for the overlearning-extinction and contrast effects, it seems necessary also to assume a disruptive, frustration-like process. We may think of it simply as a competing avoidance tendency generated as a function of discrepancy between the strength of the existing approach tendency and that which the new reinforcer (if any) is capable of supporting (cf. Daly \& Daly, 1982). There is no assumption here that the animal remembers and compares reinforcers. In the Daly model, which was designed to deal with the data for rats, the avoidance tendency is itself conditioned (Amsel's conditioned frustration), an assumption prompted by the occurrence of the various paradoxical reward effects in widely spaced trials (yesterday's frustration influencing today's performance), but the honeybee data do not require that assumption. The overlearning-extinction and contrast effects as they are found in honeybees can be understood in terms of unconditioned frustration alone, and recent experiments on partial reinforcement designed specifically to look for con- 
trol of performance by associatively reinstated effects of nonreinforcement have yielded only negative results (Ammon, Abramson, \& Bitterman, 1986). Given the possibility, at that time, of explaining the overlearningextinction effect in terms of reduced hunger and the contrast effect in terms of adaptation level, the partial reinforcement results suggested that there might be no need to assume even unconditioned frustration. The present results point again, however, to a process generated by reduction in magnitude of reinforcement that disrupts appetitive performance in honeybees.

\section{REFERENCES}

Ammon, D., Abramson, C. I., \& Bitterman, M. E. (1986). Partial reinforcement and resistance to extinction in honeybees. Animal Learning \& Behavior, 14, 232-240.

AMSEL, A. (1958). The role of frustrative nonreward in noncontinuous reward situations. Psychological Bulletin, 55, 102-119.

AMSEL, A. (1962). Frustrative nonreward in partial reinforcement and discrimination learning. Psychological Review, 69, 306-328.

Bitterman, M. E., Menzel, R., Fietz, A., \& SChÄFer, S. (1983). Classical conditioning of proboscis extension in honeybees (Apis mellifera). Journal of Comparative Psychology, 97, 107-119.

Couvillon, P. A., \& Bitterman, M. E. (1980). Some phenomena of associative learning in honeybees. Journal of Comparative \& Physiological Psychology, 94, 878-885.
Couvillon, P. A., \& BitTerman, M. E. (1984). The overlearningextinction effect and successive negative contrast in honeybees (Apis mellifera). Journal of Comparative Psychology, 98, 100-109.

DAlY, H. B., \& DALY, J. T. (1982). A mathematical model of reward and aversive nonreward: Its application in over 30 learning situations. Journal of Experimental Psychology: General, 111, 441-480.

GrossmanN, K. E. (1973). Continuous, fixed-ratio, and fixed-interval reinforcement in honey bees. Journal of the Experimental Analysis of Behavior, 20, 105-109.

Hull, C. L. (1943). Principles of behavior. New York: AppletonCentury-Crofts.

Ison, J. R., * Cook, P. E. (1964). Extinction performance as a function of incentive magnitude and number of acquisition trials. Psychonomic Science, 1, 245-246.

PERIN, C. T. (1942). Behavior potentiality as a joint function of the amount of training and the degree of hunger at the time of extinction. Journal of Experimental Psychology, 30, 93-113.

SigurDson, J. E. (1981). Automated discrete-trials techniques of appetitive conditioning in honey bees. Behavior Research Methods \& Instrumentation, 13, 1-10.

Traupmann, K. L. (1972). Drive, reward, and training parameters, and the overlearning-extinction effect (OEE). Learning \& Motivation, 3, 359-368.

(Manuscript received March 24, 1986; revision accepted for publication August 21, 1986.) 